\title{
PRAXISTHEMA
}

\section{s::can-Messstationen überwachen kontinuierlich die Qualität der Wiener Grundwasserbewirtschaftung}

\author{
DI FRANZ HOFSTÄDTER und DR. JOEP VAN DEN BROEKE, s::can Messtechnik GmbH
}

Nach österreichischem Wasserrechtsgesetz bedürfen Einwirkungen auf Gewässer, die unmittelbar oder mittelbar deren Wassergüte beeinträchtigen, jedenfalls einer wasserrechtlichen Bewilligung. Letztere wurde im Fall des Kraftwerkes Wien-Freudenau mit der Auflage eines intensiven Programms zur Beweissicherung versehen, da die Beurteilung größerer Eingriffe in den Wasserhaushalt erfahrungsgemäß die langfristige Analyse von Daten zur Hydrologie und Wasserbeschaffenheit erfordert. Neben anderen Maßnahmen betreibt die VERBUND-Austrian Hydro Power AG (AHP) deshalb im Staubereich des Kraftwerkes Wien-Freudenau sechs Messstationen, welche die Wasserqualität teils im Donauwasser, teils im Uferfiltrat kontinuierlich überwachen (vgl. auch Abb. 1). Die umfassenden Maßnahmen zur Beweissicherung vor Beginn, während und nach Abschluss der Baumaßnahmen zeigen, dass nicht nur die zu sichernde Grundwasserdynamik im Bereich des zweiten und zwanzigsten Wiener Gemeindebezirkes durch eine aktive Bewirtschaftung des Grundwassers beibehalten werden konnte, sondern dass sich darüber hinaus die Grundwassergüte verbessert hat.

Um den von der Wasserrechtsbehörde vorgeschriebenen Parameterumfang vollständig, möglichst lückenlos und in der besten Qualität zu erfassen, werden die Resultate der in den Messstationen verwendeten Online-Messgeräte regelmäßig bewertet. Darüber hinaus werden auch neu entwickelte Messtechnologien auf ihr Potenzial hin getestet, um eine Verbesserung des aktuellen Zustandes zu bewirken. Dazu wird neben der Aussagekraft von Ersatzparametern und der Anwendbarkeit alternativer Messtechnologien auch die im praktischen Messbetrieb erreichbare Verfügbarkeit und Präzision einer detaillierten Evaluierung unterzogen.

Ein wesentlicher Parameter zur Beurteilung der Qualität von Fluss- und Grundwasser ist die Konzentration der Summe an organischen Substanzen. Neben den im Gewässer biologisch (Algen,

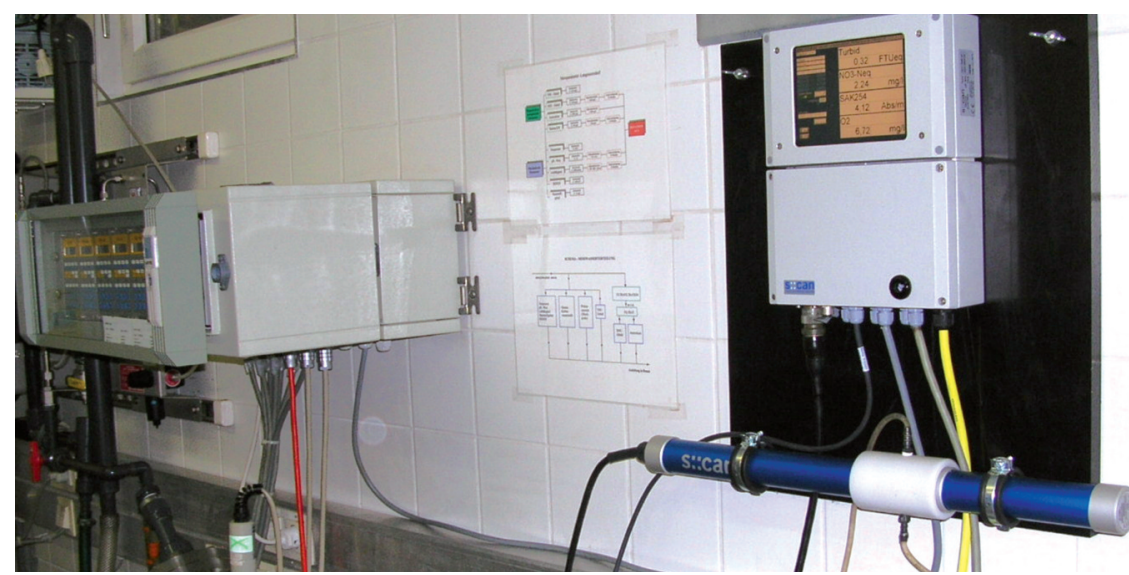

Abb. 1: Messstation der AHP mit s::can-Spektrometersonde (Fabrikat spectro::Iyser'"') zur kontinuierlichen Messung der Wasserqualität des Uferfiltrates

Bakterien) entstehenden organischen Stoffen kommt es zusätzlich zu Einträgen (Niederschlagsereignisse, Schneeschmelze etc.). Da Turbulenzen der Wasserströmung organisches Material aus den Sedimenten an der Gewässersohle in den freien Wasserkörper einbringen können, beeinflusst auch die wasserwirtschaftliche Nutzung der Donau (z. B. Schifffahrt, Wasserkraftwerke) den Gehalt an organischem Material im Flusswasser. Grundsätzlich lässt sich natürliches organisches Material (Natural Organic Matter - NOM) nach seiner Größe in partikuläres (Particular Organic Matter - POM) und gelöstes (Dissolved Organic Matter-DOM) organisches Material klassifizieren. In der chemischen Laboranalytik hat sich im Trink-, Grundund Oberflächenwasser heute fast durchgehend der Parameter TOC (Total Organic Carbon) bzw. DOC (Dissolved Organic Carbon) zur Quantifizierung des Gehaltes der Summe an organischen Kohlenstoffverbindungen durchgesetzt.

Eine alternative physikalische Methode, die sich zur kontinuierlichen Bestimmung des Gehaltes an organischen Stoffen in Echtzeit besonders gut eignet, ist die Messung der Absorption von ultraviolettem Licht. So genannte chromophore Verbindungen (z. B. Doppelbindungen), die in vielen organischen Substanzen sowohl natürlicher (z. B. Hu- minstoffen) als auch anthropogener Herkunft (z. B. Aromaten) enthalten sind, absorbieren UV-Strahlung. Obschon nicht alle existierenden organischen Verbindungen erfasst werden, besteht in den meisten Anwendungen eine sehr gute Korrelation zu im Labor ermittelten Parametern der Wasseranalytik, da eine deutliche Mehrheit der üblicherweise in Gewässern vorkommenden organischen Substanzen solche Chromophore beinhalten [1]. Sowohl alltägliche Ereignisse hoher Häufigkeit (z. B. Hochwässer), als auch außergewöhnliche Ereignisse geringer Auftrittswahrscheinlichkeit, aber gleichzeitig mit sehr hohem Schadenspotenzial (z. B. Ölunfälle), können durch die Messung der Absorption deutlich angezeigt werden.

Die Messung der Absorption bei einer Wellenlänge von $254 \mathrm{~nm}$ (SAK254 oder UV254) wurde aus technologischen Gründen vor etwa 30 Jahren zur Quantifizierung der organischen Kohlenstoffverbindungen eingeführt, weil damals verfügbare Lichtquellen bei dieser Wellenlänge ihr Emissionsmaximum aufwiesen. Chemisch-analytisch betrachtet ist eine Messung über den gesamten ultravioletten Wellenlängenbereich zur Quantifizierung der Summe an organischen Stoffen allerdings überlegen, da viele organische Stoffe ihr Absorptionsmaximum im Spektralbereich von 


\section{PRAXISTHEMA}

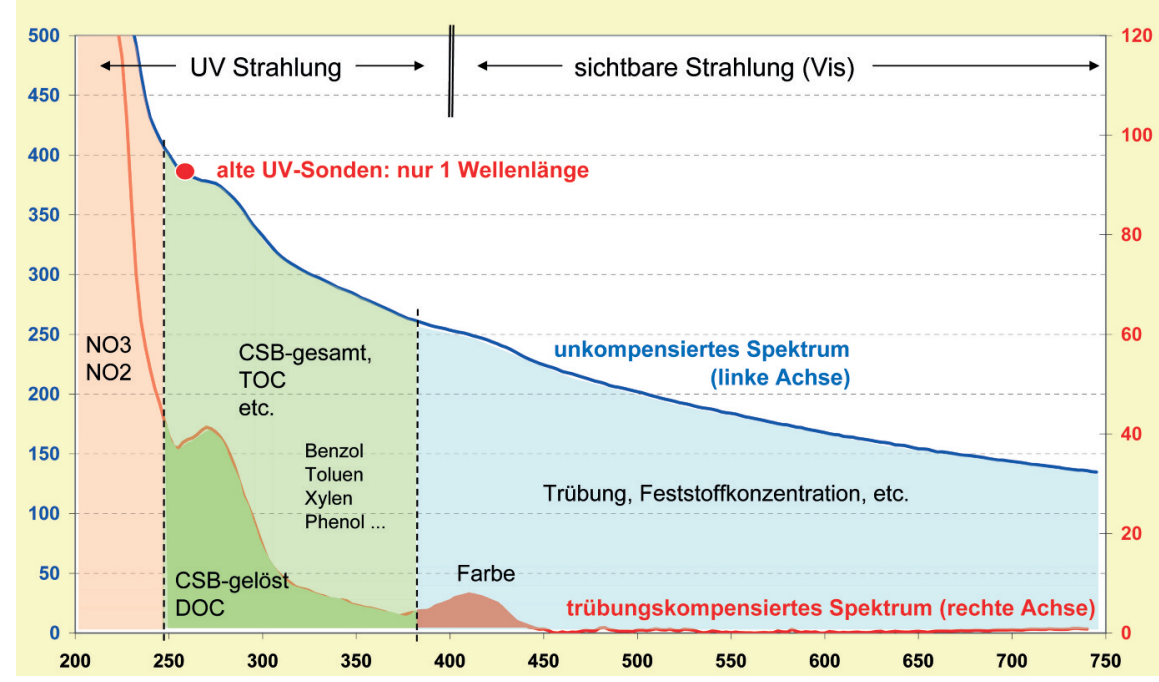

Abb. 2: Von s::can-Spektrometersonden erfasstes UV-Vis-Absorptionsspektrum, verursacht durch gelöste Substanzen (rot) bzw. alle Wasserinhaltsstoffe (blau) inkl. Angabe der zur Bestimmung verschiedener Parameter verwendeten Wellenlängenbereiche

$200 \mathrm{~nm}$ bis $400 \mathrm{~nm}$ bei anderen Wellenlängen als bei $254 \mathrm{~nm}$ haben, bzw. bei einer Wellenlänge von $254 \mathrm{~nm}$ sogar ein Absorptionsminimum aufweisen. Darüber hinaus ergeben sich auch messtechnische Vorteile, weil die UV-Absorption eines Stoffes immer einen gewissen Wellenlängenbereich einschließt, d. h. Substanzen, die auch bei $254 \mathrm{~nm}$ Strahlung absorbieren, werden auch im benachbarten Wellenlängenbereich erfasst (vgl. auch Abb. 2).

Mit der s::can-Spektrometersonde wurde vom österreichischen Unternehmen s::can Messtechnik GmbH im Jahr 1999 erstmals eine tauchfähige UV-VisSpektrometersonde in die Wassermesstechnik eingeführt, welche die spektrale Erfassung von organischen Summenparametern erlaubt. Neben den grundsätzlichen Vorzügen einer optischen Messmethode (Wartungsarmut, Betriebssicherheit, Reproduzierbarkeit etc.) erlaubt die Erfassung des kompletten UV-Vis-Absorptionsspektrums nicht nur aus oben angeführten Gründen die Online-Bestimmung von organischen Summenparametern in einer zuvor nicht bekannten Qualität (die simultane Erfassung von ebenfalls im UV-Bereich absorbierenden Teilchen erlaubt die rechnerische Kompensation der von ihnen bewirkten Interferenzen auf die Messung der UV-Absorption in Echtzeit). Der Spektrometrie sind außerdem weitere Standardparameter derWasserqualitätsmessung zugänglich (z. B. $\mathrm{NO}_{3} \mathrm{~N}$, $\mathrm{NO}_{2} \mathrm{~N}$, FTU/AFS), und sogar eine Fraktionierung der organischen Substanzen (z. B. TOC und DOC oder CSB und BSB) ist möglich (vgl. auch Abb. 2).

Im Rahmen eines europäischen Forschungsprojektes wurde 2001 die Übereinstimmung von spektralen Ersatzparametern durch die Messsonde spectro::lyser $^{\mathrm{TM}}$ der Firma s::can mit standardisierten Labormethoden zur Bestimmung der organischen Substanz umfassend untersucht. Dazu wurde der Ablauf von mit Uferfiltrat der Donau beschickten Test- filtern mittels Spektrometersonden kontinuierlich gemessen und diskrete Wasserproben für die DOC-Analyse entnommen. Die beobachtete Übereinstimmung zwischen im Labor ermitteltem DOC und spektral bestimmtem mit der s::canSpektrometersonde war sehr hoch $\left(\mathrm{R}^{2}=\right.$ 0,95). Im Vergleich zur herkömmlichen TOC- bzw. DOC-Analyse zeigten die Ergebnisse der s::can-Spektrometersonde eine bessere Messgenauigkeit [4]. Auch bei der Messung des wichtigen Nährstoffparameters Nitrat mit der s::canSpektrometersonde zeigte sich eine gute Übereinstimmung zu den Laborergebnissen. In der untenstehenden Abb. 3 sind exemplarisch Nitratresultate von Messungen in verschiedensten Oberflächengewässern dargestellt: Die Korrelationen im linken Diagramm - basierend auf einer so genannten „Globalen Kalibration" von s::can - sind eindeutig besser als jene im rechten unterVerwendung einzelner Wellenlängen. Da s::can-Spektrometersonden „Globale Kalibrationen“ benutzen, die auf vielen Wellenlängen des Absorptionsspektrums basieren, können Querempfindlichkeiten sehr gut kompensiert werden. Einfache Photometersonden, die nur einzelne Wellenlängen verwenden, erreichen deshalb schlechtere Korrelationen zu Resultaten konventioneller Labormethoden.

In Abstimmung mit der Obersten Wasserrechtsbehörde wurden im Auftrag der AHP zwei umfassende Studien erstellt [2, 3], welche die im Zeitraum Juli 2001 bis Juni 2004 im Brunnen 21 erfassten Messergebnisse statistisch analysierten, mit dem Ziel, die Betriebssicherheit der kontinuierlichen Wasserqualitätsmessung zu erhöhen. Aus Gründen der Vergleichbarkeit mit an dieser Messstelle vor 2001 durch eine einfache Photometersonde erfassten Zeitreihen wurde das Hauptaugenmerk der statistischen Analyse auf den Parameter SAK254 gelegt.
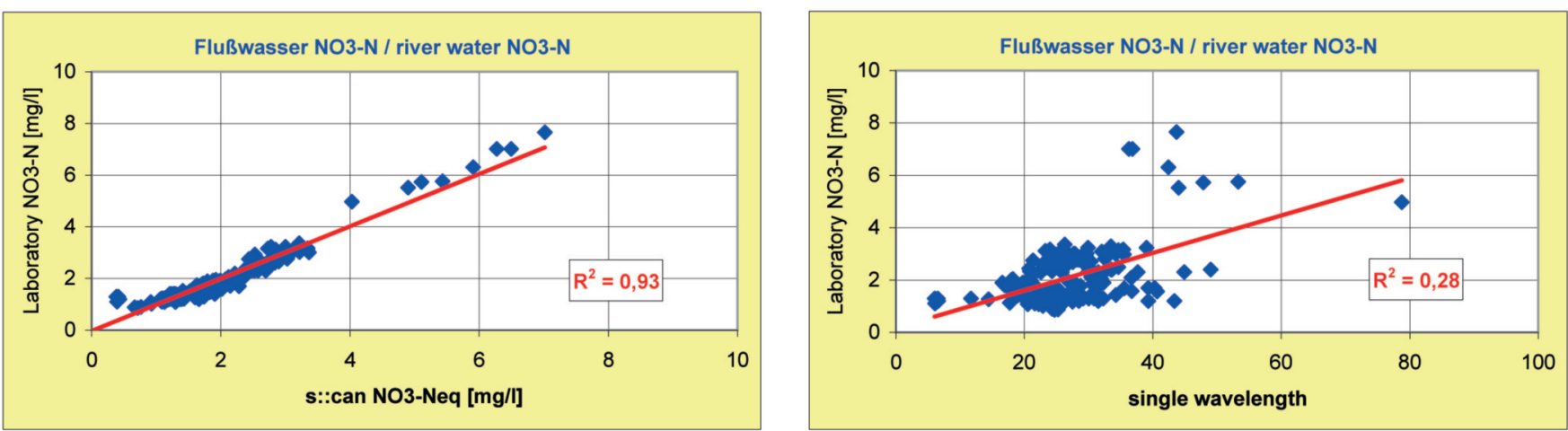

Abb. 3: Bessere Korrelationen zu Laborergebnissen unter Verwendung der „Globalen Kalibration“ für Flusswasser (links) als unter Verwendung einzelner Wellenlängen (rechts) am Beispiel von Nitratmessungen im Flusswasser 


$\begin{array}{lcccc}\text { Gerät } & \text { Parameter } & \begin{array}{c}\text { Theoretische Maximalzahl } \\ \text { Halbstundenwerte }\end{array} & \begin{array}{c}\text { Anzahl } \\ \text { Halbstundenwerte }\end{array} & \begin{array}{c}\text { Verfügbarkeit } \\ \text { Halbstundenwerte }\end{array} \\ \text { s::can Spektrometersonde } & \text { SAK254 } & 51815 & 50149 & 97 \% \\ \text { Analysatorschrank } & \text { DOC } & 51815 & 38779 & 75 \%\end{array}$

Tab. 1: Gegenüberstellung der Verfügbarkeit der s::can-Spektrometersonde und jener eines parallel betriebenen Analysatorschrankes im Zeitraum 15. Mai 2001 bis 14 . Mai 2004

Es ergaben sich signifikante Korrelationen zwischen dem mittels Schrankanalysator erfassten Parameter DOC und dem mittels Spektrometersonde (Fabrikat uv::lyser ${ }^{\mathrm{TM}}$ der Firma s::can) gemessenen Parameter SAK254. Obschon die organischen Parameter DOC und SAK254 im Uferfiltrat vom Durchfluss in der Donau dominiert werden (sh. Abb. 4), zeigen sie keinen direkten Zusammenhang mit dem Wasserstand. Beide Parameter erreichen in der Zeit geringerer Durchflusseinwirkung einen sehr hohen Grad des Zusammenhanges $(\mathrm{R}>0,90)$. Da beide Parameter den $\mathrm{Ge}$ halt an organischen Substanzen im Uferfiltrat zeigen, ist ein Ersatz des Parameters DOC durch den Parameter SAK254 möglich [2].

Da etwa zwei Drittel aller bewirtschaftungsrelevanten Störungen bei der Grundwasserbewirtschaftung durch das DOC-Analysegerät bedingt werden, ist es erklärtes Ziel der AHP, nach Möglichkeit die Messung mittels Schrankanalysatoren schrittweise durch eine kontinuierliche Messung mittels Spektrometersonden (Fabrikat spectro::lyser ${ }^{\mathrm{TM}}$ bzw. uv::lyser ${ }^{\mathrm{TM}}$ der Firma s::can) zu ersetzen. Die durchgehende Verwendung von s::can-Spektrometersonden kann die Ausfälle der Grundwasserbewirtschaftung deutlich verringern [2].

Die Geräteanfälligkeit wurde durch die Auswertung der Verfügbarkeit beider Geräte in einem Datenzeitraum von mehr als drei Jahren (sh. Tab. 1) in der Messstation Brunnen 21 beurteilt. Während vom DOC-Analysegerät nur etwa $75 \%$ der theoretisch möglichen Werte vorhanden sind, stellt die s::can-Spektrometersonde unter Berücksichtigung einer zweiwöchigen Reparatur etwa $97 \%$ der möglichen Werte zur Verfügung. Somit ist die s::can-Spektrometersonde bedeutend betriebssicherer als das DOCAnalysegerät [2].

Darüber hinaus zeigt eine detaillierte Betrachtung der in Echtzeit erfassten Messwerte beeindruckend die "methodenbedingt" bessere Präzision der von der s::can-Spektrometersonde erfassten Messwerte (vgl. Abb. 5). Die Resultate der s::can-Spektrometersonde (blau) zeigen Veränderungen der Wasserqualität, die zeitlich mit Änderungen des Pegel-

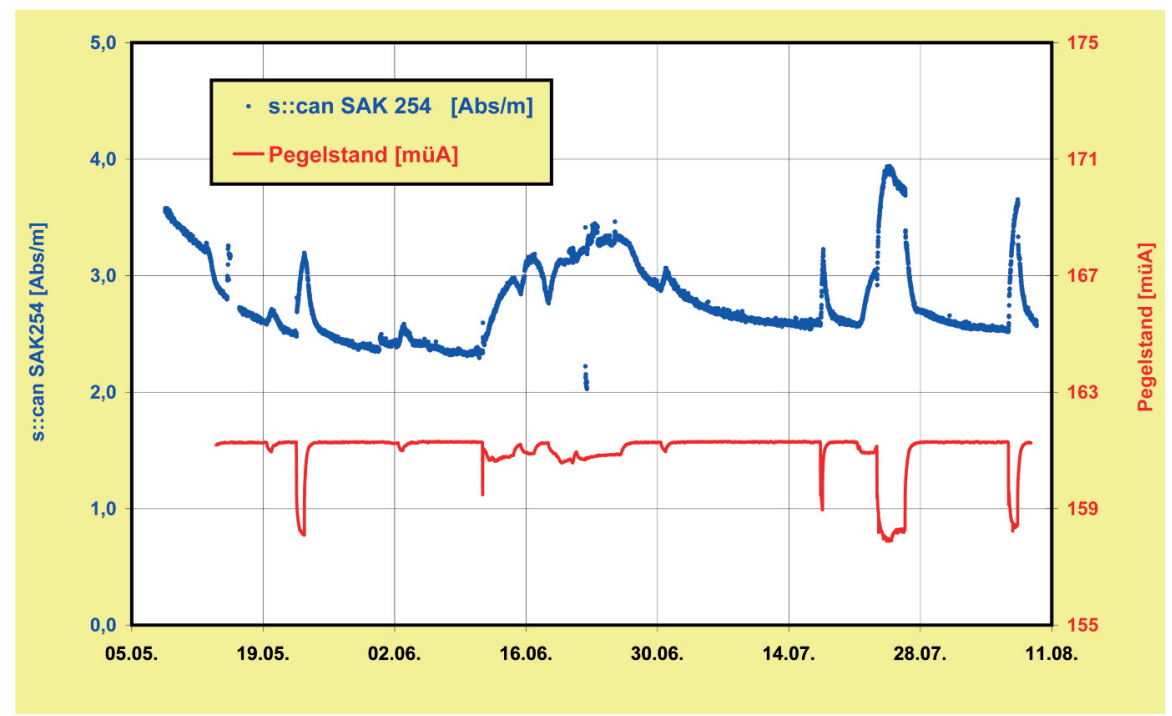

Abb. 4: Mittels s::can-Spektrometersonde kontinuierlich erfasste Zeitreihe SAK254 (blau) und Pegelmessung (rot) im Brunnen 21

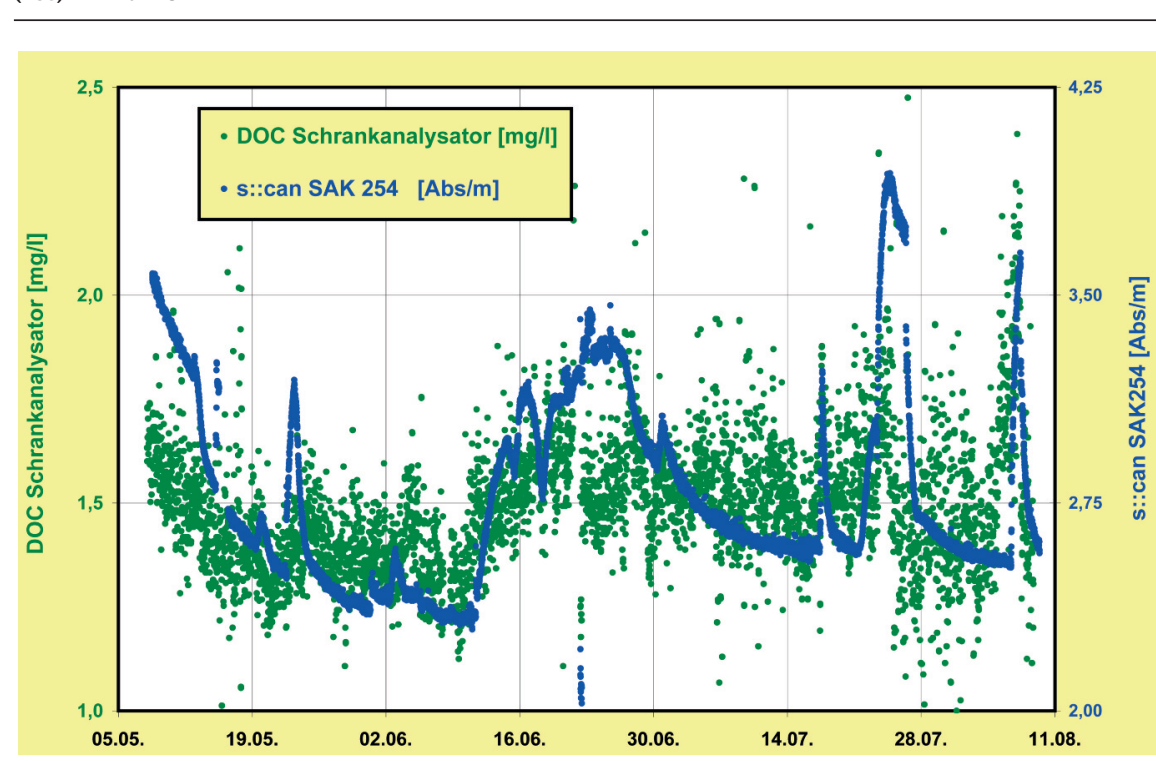

Abb. 5: Gegenüberstellung der mittels s::can-Spektrometersonde kontinuierlich erfassten Zeitreihe SAK254 (blau) mit der Zeitreihe eines parallel betriebenen Analysatorschrankes (grün)

standes zusammenfallen, und demnach durch die unterschiedliche Wasserführung der Donau verursacht werden. Dieselben Ereignisse verschwinden im Messrauschen der Resultate des DOCAnalysatorschrankes (grün) und sind dort keiner inhaltlichen Interpretation zugänglich.

Mittlerweile werden von der AHP an verschiedenen Messstationen vier s::can-Spektrometersonden (Fabrikate uv::lyser ${ }^{\mathrm{TM}}$ und spectro::lyser ${ }^{\mathrm{TM}}$ ) betrie- ben, teilweise zur kontinuierlichen Messung der Wasserqualität der Donau, teilweise zur Überwachung der Qualität des Uferfiltrates. Auch im unfiltrierten Donauwasser werden vergleichbare Resultate, wie oben dargestellt, erzielt, was nur durch die Verwendung der automatischen Reinigung mittels Druckluft und den Verzicht auf jegliche bewegte Bauteile im Wasser möglich wird. Die eingesetzten Bedienrechner (Fabrikat con::stat der Firma s::can) erlauben auch 


\section{PRAXISTHEMA}

die digitale Integration (ModbusRTU/ RS485) anderer s:::can-Messgeräte (z. B. zur Messung der Ammoniumkonzentration, des $\mathrm{pH}$ oder der elektrischen Leitfähigkeit), sodass komplette Messstationen zur kontinuierlichen Qualitätsüberwachung aus einer Hand geliefert werden können (sh. Abb. 6). An der Messstation Nußdorf wird diese Option bereits von der AHP genutzt und eine s::can oxi::lyser ${ }^{\mathrm{TM}}$-Sauerstoffsonde in Kombination mit einer s::can-Spektrometersonde betrieben.

Durch die Anwendung der innovativen Methode der „Deltaspektrometrie“ und durch die Auswertung von Differentialspektren können s::can-Spektrometersonden auch kleinste Veränderungen im Absorptionsspektrum in Echtzeit detektieren. Damit können nach einer Anpassung an die individuelle Wassermatrix auch geringste spektrale Abweichungen der Wasserqualität vom „Normalzustand" messstellenspezifisch registriert werden. Diese Möglichkeit wird insbesondere in Frühwarnsystemen der Trinkwasserversorgung zur Detektion außergewöhnlicher Ereignisse in der Rohwasserqualität (z. B. durch das Auftreten von Pestiziden oder Aromaten) genutzt $[5,6]$.

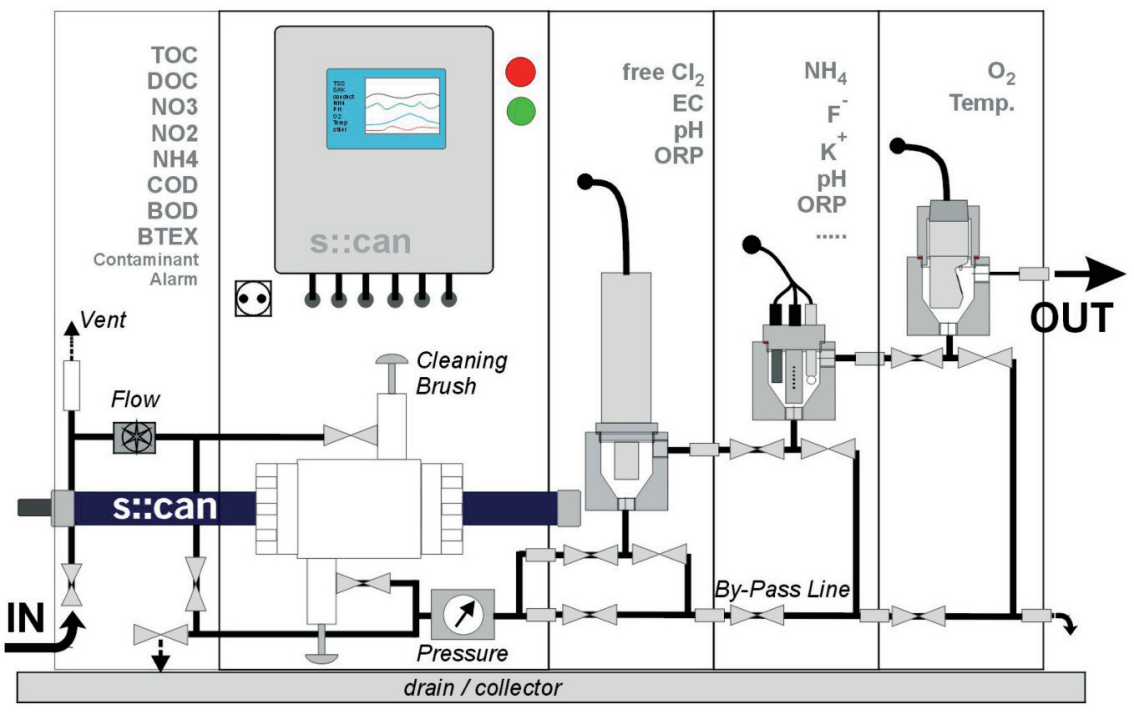

Abb. 6: Kompakte s::can Messstation zur kontinuierlichen Messung der Wasserqualität

\section{LITERATUR}

[1] Wang, G.-S.; Hsieh, S.-T. (2001): Monitoring natural organic matter in water with scanning spectrophotometer, Environment International, 26 (4), p. 205-212 [2] Weilguni, H.: Bericht „Grundwasserbewirtschaftung Brunnen 21 - Vergleich DOC und SAK254", Verbundplan $\mathrm{GmbH}$

[3] Weilguni, H.: Ergänzungsbericht „Grundwasserbewirtschaftung Brunnen 21-Vergleich DOC und SAK254 Ergänzende Untersuchungen “, Verbundplan $\mathrm{GmbH}$ [4] Fleischmann, N.; Werderitsch, M.; Weyermayr, H.: Management of sensible water uses with the help of innovative sensor technology - Part 2: Testfilter results, Proceedings der IWA-Konferenz „Automation in Water Quality Monitoring“, Wien, 21.-22. Mai 2002 [5] van den Broeke, J.; Brandt, A.; Hofstädter, F.; Weingartner, A. (2006): Monitoring of Organic Micro Contaminants in Drinking Water using a Submersible UV/Vis Spectrophotometer, Proceedings of the NATO Advanced Research Workshop on Security ter, Croatia, 27.-31. Mai 2005, Pollert, J.; Dedus, B. (Eds.), Springer Verlag, Dordrecht, p. 19-29 [6] Langergraber, G.; Weingartner, A.; Fleischmann, N. (2004): Time resolved delta spectrometry: a method to define alarm parameters from spectral data, Water Science and Technology, 50 (11), p. 13-20

Informationen:

:.can Messtechnik GmbH DI Franz Hofstädter Dr. Joep van den Broeke Brigittagasse 22-24 1200 Wien

Tel. +43-1-219 7393 Fax +43-1-219 73 93-12

sales@s-can.at www.s-can.at of Water Supply Systems: From Source to Tap, Mur- 